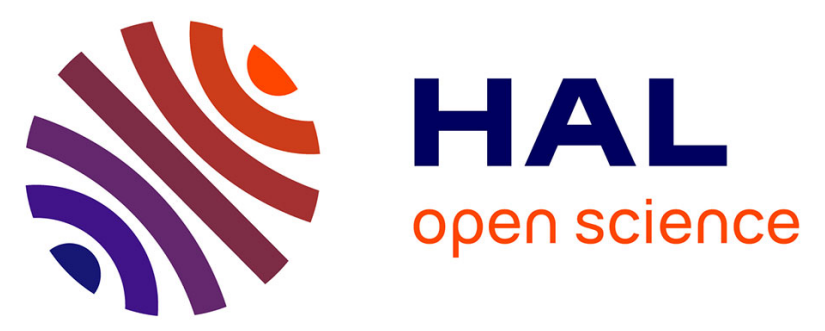

\title{
Transmission-Reflection Analysis in high scattering optical fibers: A comparison with single-mode optical fiber
}

Mariana Silveira, Anselmo Frizera, Arnaldo Leal-Junior, Diana Ribeiro, Carlos Marques, Wilfried Blanc, Camilo A.R. Diaz

\section{To cite this version:}

Mariana Silveira, Anselmo Frizera, Arnaldo Leal-Junior, Diana Ribeiro, Carlos Marques, et al.. Transmission-Reflection Analysis in high scattering optical fibers: A comparison with single-mode optical fiber. Optical Fiber Technology, 2020, 58 (16), pp.102303. 10.1016/j.yofte.2020.102303 . hal-03105406

\section{HAL Id: hal-03105406 \\ https://hal.science/hal-03105406}

Submitted on 11 Jan 2021

HAL is a multi-disciplinary open access archive for the deposit and dissemination of scientific research documents, whether they are published or not. The documents may come from teaching and research institutions in France or abroad, or from public or private research centers.
L'archive ouverte pluridisciplinaire $\mathbf{H A L}$, est destinée au dépôt et à la diffusion de documents scientifiques de niveau recherche, publiés ou non, émanant des établissements d'enseignement et de recherche français ou étrangers, des laboratoires publics ou privés. 


\title{
Transmission-Reflection Analysis in high scattering optical fibers: A comparison with single-mode optical fiber
}

\author{
Mariana Silveira $^{\mathrm{a}}$, Anselmo Frizera ${ }^{\mathrm{a}}$, Arnaldo Leal-Junior ${ }^{\mathrm{b}}$, Diana Ribeiro ${ }^{\mathrm{c}}$, Carlos Marques ${ }^{\mathrm{c}}$, \\ Wilfried Blanc ${ }^{\mathrm{d}}$, Camilo A. R. Díaz ${ }^{\mathrm{a}, *}$ \\ ${ }^{a}$ Telecommunications Laboratory LABTEL, Graduate Program in Electrical Engineering, Federal University of Espírito Santo, $29075-910$ Espírito Santo, Brazil \\ ${ }^{\mathrm{b}}$ Mechanical Engineering Department, Federal University of Espírito Santo, 29075-910 Espírito Santo, Brazil \\ ${ }^{\mathrm{c}}$ I3N \& Physics Department, University of Aveiro, 3810-193 Aveiro, Portugal \\ ${ }^{\mathrm{d}}$ Université Côte d'Azur, Institut de Physique de Nice - INPHYNI UMR7010, 06108 Nice Cedex 2, France
}

\section{A R T I C L E I N F O}

\section{Keywords:}

Distributed optical fiber sensors

Transmission-Reflection Analysis

Rayleigh scattering

Optical power-based sensors

Interrogation systems

\begin{abstract}
A B S T R A C T
Transmission-Reflection Analysis (TRA) is an inexpensive technique used in distributed monitoring systems for single perturbation tracking. However, TRA is limited to long distance applications $(\sim \mathrm{km})$, since it has poor spatial resolution $(\sim \mathrm{m})$ when combined to standard silica fibers. This paper proposes the use of a magnesium and erbium co-doped optical fiber in conjunction with the TRA technique. The goal is to improve spatial resolution and accuracy for detection of loss-inducing disturbances along the sensing fiber. Bending losses are induced at several points of the fiber spaced at $50 \mathrm{~cm}$ over a $5.4 \mathrm{~m}$ fiber length, where each curve is clearly identified with a maximum hysteresis of $1.65 \%$. The localization of a strong disturbance with an estimated accuracy of $\pm 0.8 \mathrm{~mm}$ near the source end and $\pm 7.2 \mathrm{~mm}$ near the fiber end is analytically demonstrated. Regarding weak disturbances, the proposed approach is vulnerable to noisy measurements. However, the response accuracy has been estimated up to $\pm 36.7 \mathrm{~mm}$.
\end{abstract}

\section{Introduction}

Distributed optical fiber sensors (DOFSs) have been widely explored since the 1980s $[1,2]$ due to their advantages over standard electronic technology, such as immunity to electromagnetic interference, galvanic isolation, resistance to chemical corrosion, lightweight and small size $[3,4]$. Furthermore, DOFSs provide the spatial distribution of the measurand along the entire length of the fiber [5], whereas electronic technology combines various single point sensors, which can be complex and costly to deploy when short sampling spans and vast monitoring areas are demanded [6]. Therefore, DOFSs have found applications in multiple fields, such as structural health monitoring $[7,8]$, industrial monitoring [9,10] and aerospace applications [11].

Distributed optical fiber loss sensors are a well established approach to locate loss-inducing perturbations [12]. Their reported applications vary from monitoring mechanical events, such as structural deformations [13], to detecting hazardous conditions, such as pipe leakages, fires and explosions $[14,15]$. The region where the loss-inducing disturbance occurs along the sensing fiber is often determined by means of two main techniques: the Optical Time Domain Reflectometry (OTDR) [16] and the Optical Frequency Domain Reflectometry (OFDR) [17].
However, both methods employ modulated light sources, which are costly [18]. In addition, they require fast electronics in order to achieve high resolution, demanding interrogation systems that are more complex and costly as compared with interrogation setups based on optical power monitoring $[19,20]$. Moreover, since many optical and electronic devices are required, OTDR and OFDR systems do not have compact size, which makes them unsuitable for wearable systems [20].

Alternatively, Transmission-Reflection Analysis (TRA) is a simpler and less expensive method developed for single perturbation tracking [21]. This technique is based on directly measuring the transmitted and backscattered optical powers of an unmodulated continuous wave light source. Unlike the OFDR method, which provides high spatial resolution $(\sim \mathrm{mm})$ [22], the TRA method has poor spatial resolution $(\sim \mathrm{m})$ when applied to standard silica fibers [18]. This sensitivity constraint is due to the low Rayleigh backscattering signal at the optical power detector [23]. In order to increase the reflected power, multiple fiber Bragg gratings (FBGs) can be inscribed into the fiber core, leading to higher spatial resolution [15,21]. However, such schemes may affect the sensor's robustness, especially if the fabrication process yields noncoated FBGs. Although the inscription of Bragg gratings in different materials is becoming less complex over the years [24], the most

\footnotetext{
* Corresponding author.

E-mail address: c.rodriguez.2016@ieee.org (C.A. R. Díaz).
} 
widespread method, i.e. phase mask technique, requires complex optical equipment, such as UV laser, phase mask, optical lens and kinematic mirrors, that need to be precisely assembled $[25,26]$.

Another way of enhancing the reflected optical power is to use high scattering medium, such as silica optical fibers containing oxide nanoparticles. Among the procedures for creating nanoparticles, one strongly emphasized in the recent literature consists of using an alkaline-earth salt, e.g. magnesium ( $\mathrm{Mg}$ ) and calcium (Ca), as a phase-separating agent [27]. During the fiber fabrication process, the silicabased preform is soaked with a doping solution containing alkalineearth ions, resulting in an immiscible system. This system decomposes into two phases, one silica-rich and one rich in alkaline-earth nanoparticles. The presence of nanoparticles in the fiber core leads to higher optical losses due to the Rayleigh scattering [28]. High-scattering MgOdoped fibers obtained by the phase separation mechanism have been used as a key element to multiplex DOFSs by means of an optical backscatter reflectometer (OBR). This scheme was applied to strain, temperature and 3D shape sensing [29,30]. Additionally, MgO-doped fibers were essential to the development of OBR-based refractive index sensors with simplified fabrication $[31,32]$. These recent applications of optical fibers with oxide nanoparticles in distributed sensing systems based on backscattered power monitoring motivate efforts for enhancing the spatial resolution of TRA-based systems, since they are less expensive, portable, and have potential to achieve high acquisition rates for dynamic applications as compared with OTDR, OFDR and OBR based interrogation systems $[19,29]$.

This paper presents experimental evidence that inexpensive DOFSs can be produced by combining TRA technique with a magnesium and erbium co-doped fiber (hereinafter referred to as "NPF"). The proposed solution is compared to the conventional TRA-based sensor, which employs a standard single-mode fiber (SSMF). The remainder of this paper is organized as follows: Section 2 presents a brief theoretical background in TRA and discusses the fabrication process of the NPF as well as its main optical properties; Section 3 presents the setup for experimental characterization of the NPF sensor; the experimental results are discussed in Section 4; finally, Section 5 presents the conclusions.

\section{Theoretical background}

\subsection{TRA for single mode optical fiber}

The TRA method was first introduced by Spirin et al. [21]. This technique is based on the relationship between the transmitted and Rayleigh backscattered powers, since it uniquely depends on the position of the disturbance along the fiber. Indeed, considering equal losses, the Rayleigh backscattered power is strongly associated to the region where the disturbance occurred: the closer to the source-end, the lower the scattering power. In contrast, identical disturbances equally decrease the transmitted power regardless of its location [33]. A brief overview of TRA is provided to describe its main concept.

Considering a continuous wave signal launched into an undisturbed SSMF, the maximum power transmission $T$ and reflection $R$ coefficients are [14],

$T_{\max }=e^{-\alpha L}$

$R_{\max }=\frac{S_{\alpha}\left(1-e^{-2 \alpha L}\right)}{2}$,

where $L$ is the fiber length, $\alpha$ is its total attenuation coefficient and $S_{\alpha}$ is defined as,

$S_{\alpha}=\frac{b\left(n_{1}^{2}-n_{2}^{2}\right)}{n_{1}^{2}} \frac{\alpha_{s}}{2 \alpha}$

Here, $\mathrm{b}$ is associated with the waveguide property of the fiber and usually varies from 0.21 to 0.24 for single mode step-index fiber, $n_{1}$ and $n_{2}$ are refractive indices of the fiber core and cladding, respectively, and $\alpha_{s}$ is the attenuation coefficient due to Rayleigh scattering.

Let us consider that the fiber is affected by a loss-inducing disturbance located $l_{i}$ meters away from the fiber head (the fiber edge in which the source optical signal is launched). Assuming the normalized transmitted coefficient to be $T_{n o r m}=T / T_{\max }$, it can be proved that the normalized backscattered coefficient $R_{\text {norm }}$ is given by [33],

$$
\begin{aligned}
R_{\text {norm }}\left(T_{\text {norm }}\right) & =\frac{R}{R_{\max }} \\
& =\frac{S_{\alpha}+r_{1}-T_{\text {norm }}^{2}\left(S_{\alpha}-r_{2}\right) e^{-2 \alpha L}-S_{\alpha}\left(1-T_{n o r m}^{2}\right) e^{-2 \alpha l_{i}}}{S_{\alpha}+r_{1}-\left(S_{\alpha}-r_{2}\right) e^{-2 \alpha L}},
\end{aligned}
$$

where $r_{1}$ and $r_{2}$ are the power reflection coefficients from the fiber head and remote end, respectively. Both non-normalized transmitted $T$ and backscattered $R$ powers can be found by replacing $L$ with $l_{i}$ in Eqs. (1) and (2). Eq. (4) can be rewritten to express the position of the lossinducing disturbance along the sensing fiber, leading to:

$l_{i}\left(T_{\text {norm }}, R_{\text {norm }}\right)=\frac{1}{2 \alpha} \ln \left\{\frac{S_{\alpha}}{\left(S_{\alpha}+r_{1}\right) \frac{\partial R_{\text {norm }}}{\partial\left(T_{\text {norm }}^{2}\right)}+\left(r_{2}-S_{\alpha}\right) e^{-2 \alpha L}\left[\frac{\partial R_{\text {norm }}}{\partial\left(T_{\text {norm }}^{2}\right)}-1\right]}\right\}$

Thus, once the normalized transmitted $T_{\text {norm }}$ and backscattered $R_{\text {norm }}$ powers were experimentally measured, the position of the disturbance can be found by Eq. (5).

\subsection{Magnesium and erbium co-doped optical fiber}

During the NPF fabrication process, which can be found in Blanc et al. [34], magnesium and erbium ions are embedded within the fiber's core. In addition, small amounts of germanium and phosphorus are added in order to increase the core refractive index. Throughout this process, amorphous nanoparticles are grown in a silica preform. After the fiber drawing process, the nanoparticles evolve to their final form.

Recent observations have shown that these nanoparticles have different size, shape and composition. Similar fabrication processes led to nanoparticles within a $40-160 \mathrm{~nm}$ range in diameter [35]. Moreover, several shape patterns were identified, such as spheres, cylinders and irregular forms with different diameters along their lengths [36]. In addition, evidences suggest that the concentration percentage of magnesium and erbium starts increasing at a predefined radius [35]: the composition of smaller nanoparticles remains constant with size, however it starts to change if the radio surpasses a critical value.

As the nanoparticle refractive index depends on its size and composition, the aforementioned features hinder the understanding of light scattering in such medium [37]. In addition, big-sized nanoparticles induce light interferences, since the "independent scattering regime" becomes invalid as the particles fraction volume increases [36]. For these reasons, considerations made by the conventional TRA model, which was developed for SSMFs, are no longer valid. These considerations include neglecting multiple internal reflections and assuming that the refractive index and attenuation coefficient are maintained constant. Hereinafter, an experimental characterization of the NPF-based TRA sensor is provided.

\section{Materials and methods}

\subsection{Setup for experimental characterization}

The schematic of the proposed system is depicted in Fig. 1. The light emitted by a super-luminescent diode centered at $1550 \mathrm{~nm}$ with a bandwidth of $60 \mathrm{~nm}$ (SLED, DL-BP1-1501A, Ibsen Photonics, Farum, Denmark) was launched into the fiber under test (FUT) through a 99/1 optical splitter. The launched power was about $10 \mathrm{~mW}$ and $1 \%$ of this 


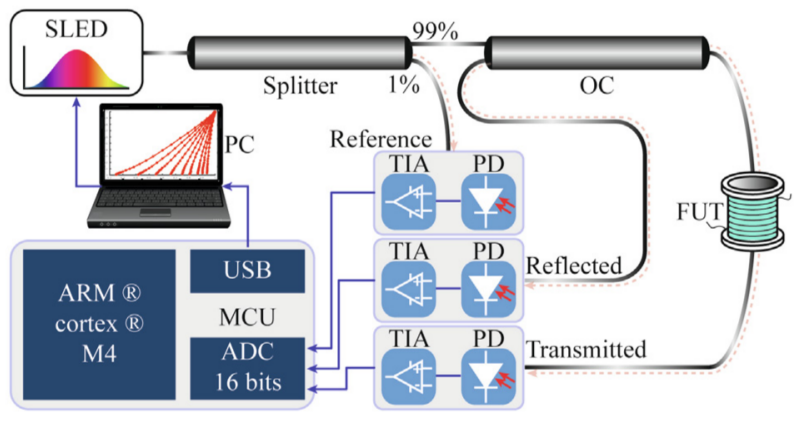

Fig. 1. Schematic of the proposed system.

power was monitored into order to identify light source fluctuations. The remaining source power was launched into an optical circulator (OC), which enabled measuring the transmitted and backscattered powers from the FUT. The experiments were conducted with two different fibers, namely the NPF and the SSMF, of equal lengths of $5.4 \mathrm{~m}$. Each optical signal was converted to an electrical signal by means of a photodetector (PD, GT322D, Go4fiber, Hong Kong, China) coupled to a transimpedance amplifier (TIA, TLV3541, Texas Instruments, USA). The signal acquisition was performed by a microcontroller unit (MCU) featuring an $\mathrm{ARM}^{\circledast}$ Cortex $^{\circledR}$-M4 core running up to $120 \mathrm{MHz}$ (Kinetis K64F, NXP Semiconductors, Eindhoven, Netherlands). The MCU has an on-chip analog-to-digital converter (ADC) with 16 bits of resolution. The acquired data was transmitted to a computer ( $\mathrm{PC}$ ) for data processing.

To create a controlled system for inducing bending losses, a plastic structure was 3D printed and anchored to a linear translation platform as depicted in Fig. 2. The printed plastic structure is composed of two main parts that can be categorized as fixed and moveable. The fixed part is attached to the external support of the linear translation platform and contains a compartment for allocating a 46-mm-length fiber segment. The moveable part, which is anchored on the translation stage, moves toward compressing this fiber segment with adjustable intensity. As the axial displacement of the translation stage is controlled by means of a micrometric manual control, the intensity of the compression process can be easily managed during experimental tests. In order to induce excess losses, five 5-mm-diameter pipes were spaced $10.2 \mathrm{~mm}$ apart and glued on the fixed and moveable parts. The inset in Fig. 2 shows the top view of the aforementioned structure (hereinafter referred as "bending station") completely closed (maximum induced bending loss).

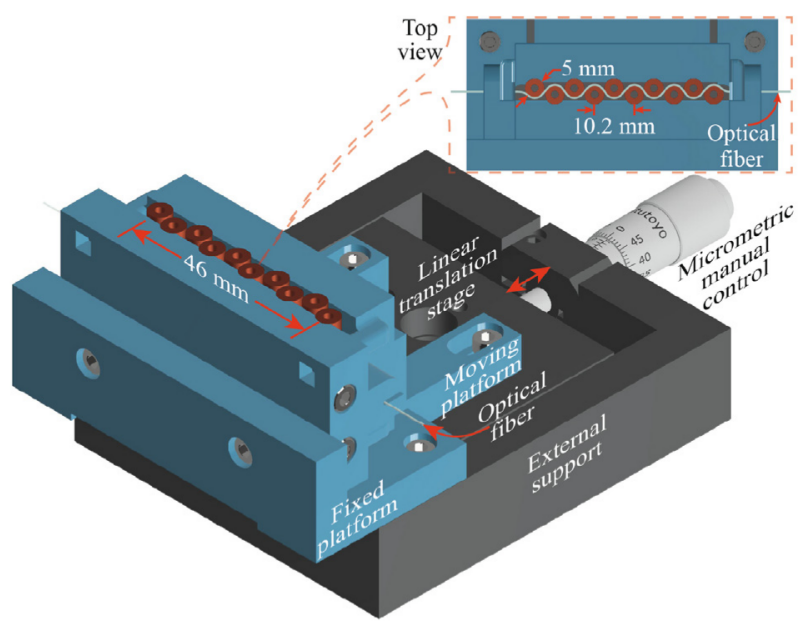

Fig. 2. Bending station based on a linear translation stage.

\subsection{Performed experiments}

To examine the behaviour of both transmitted and backscattered powers under different input power levels, the source output power was gradually raised from 0 to $10 \mathrm{~mW}$, in steps of $1 \mathrm{~mW}$. Meanwhile, the transmitted, backscattered and source reference powers were measured along time. No bending losses were induced at any region of the FUT.

In order to analyze the sensor response, the input optical power was kept constant and bending losses were induced one at a time at different regions located $l_{i}=i \times \Delta L$ meters from the fiber head, where $\Delta L$ is the spatial displacement between consecutive measurements and $i=1,2,3 \ldots$. Two values of $\Delta L$ were experimentally analyzed, namely $0.5 \mathrm{~m}$ and $0.15 \mathrm{~m}$. In each distance $l_{i}$, losses were gradually increased through the bending station, until maximum loss condition $\left(T_{n o r m} \sim 0\right)$ was reached. At the beginning of this procedure, the bending station's moveable and fixed parts were set apart $6 \mathrm{~mm}$ from each other, leaving the fiber under an undisturbed condition. Afterward, the micrometric manual control was increased in steps of $5 \mu \mathrm{m}$, until the bending stage closed completely. After reaching maximum loss condition, bending losses were decreased with the purpose of analyzing hysteresis.

It is worth mentioning that, during both experiments, measurements from the proposed data acquisition system were compared to measurements from an optical power meter (OPM, CMA5, Anritsu, Tokyo, Japan) and there was no evidence that the electronic components represented in Fig. 1 introduced nonlinearities in data.

\section{Results and discussion}

\subsection{Optical source power variations}

Fig. 3 depicts the transmitted (dash-dot line with round markers) and backscattered (dashed-line with square markers) powers from the NPF as a function of the source output optical power. The reference signal (solid line with diamond markers) represents $1 \%$ of the total source power (see Fig. 1). Once the optical signals were digitally converted, they are displayed in Fig. 3 as electric signals measured in volts. Source fluctuations were mitigated by subtracting the transmitted and backscattered signals by the reference. Regarding Fig. 3, the transmitted and reflected powers showed non-linear behaviors. Therefore, it is possible to conclude that the NPF's attenuation coefficient depends on the launched input power. As discussed before, this may be explained by the fact that the nanoparticles in the fiber's core have different dimensions (their size distribution varies between tens to hundreds of nanometers), shapes and compositions leading to nonlinearities.

For comparison purposes, Fig. 4 presents the transmitted and

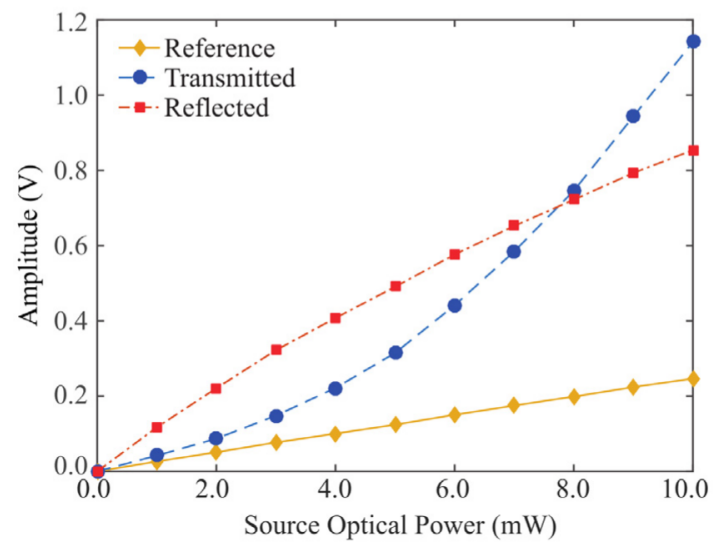

Fig. 3. Transmitted (dash-dot line with round markers), backscattered (dashedline with square markers) and reference (solid line with diamond markers) powers from the NPF for different input power levels. 


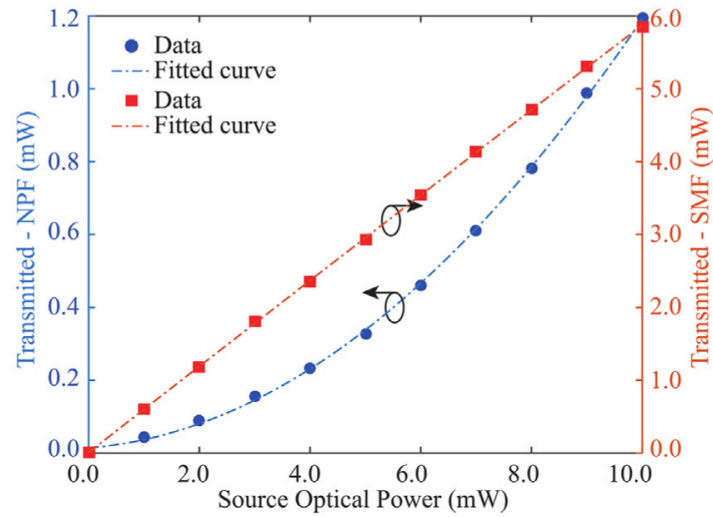

(a)

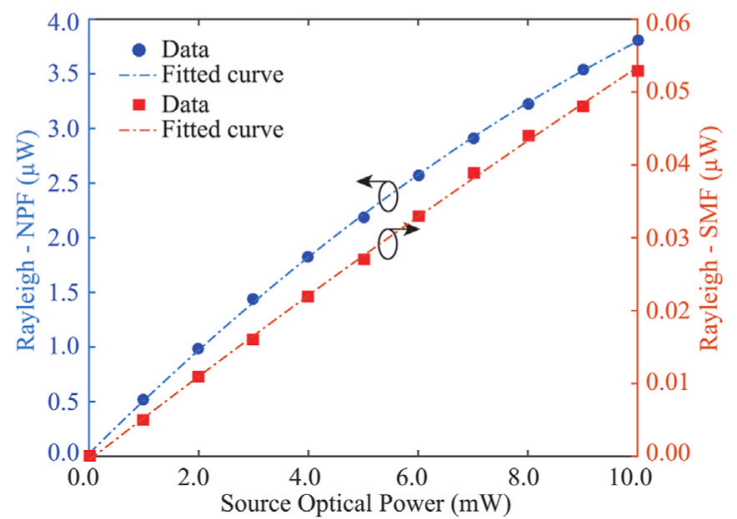

(b)

Fig. 4. Transmitted (a) and backscattered (b) powers from NPF and SSMF for different input power levels.

backscattered powers from the NPF and SSMF obtained under the aforementioned circumstance of different input optical power levels. The measurands were converted into power. As expected, the power transmitted through the SSMF's core increased linearly in accordance with the source output power (Fig. 4a, dashed line with square markers). It is worth noting that the transmitted power is higher than that measured from the NPF. This comes from the lower attenuation of SSMFs $(\sim 0.2 \mathrm{~dB} / \mathrm{km})$. The backscattered power of both fibers are presented in Fig. 4b. The presence of special nanoparticles increases the Rayleigh backscattered power by more than $700 \%$ as compared with the SSMF. This feature suggests that NPF-based TRA sensors may be used for short distance applications.

\subsection{Distributed disturbance analysis}

Fig. 5 depicts the relationship between the normalized transmitted $\left(T_{\text {norm }}\right)$ and backscattered $\left(R_{\text {norm }}\right)$ powers for bending losses induced along the FUT. The measurands displayed in Fig. 5 were normalized with respect to their initial undisturbed values. However, by completely closing the bending station, the transmitted power changed by more than $-20 \mathrm{~dB}(\mathrm{NPF})$ and $-10 \mathrm{~dB}$ (SSMF) from its initial undisturbed state. The results of the NPF are presented in Fig. 5a. Solid lines without markers depict the measurements acquired assuming $\Delta L=0.5 \mathrm{~m}, i=1,2, \ldots, 9$ and, as a consequence, $l_{i}=0.5,1.0,1.5, \ldots, 4.5 \mathrm{~m}$. Each point located between the extremes curves $l_{1}$ and $l_{9}$ is uniquely related to one parametric curve that crosses this point. Therefore, after previous characterization, the position of the disturbance may be estimated by discovering which parametric curve is closer to the point with coordinates equal to the measured normalized Rayleigh backscattered and transmitted powers. Hysteresis in measurements was calculated by Eq. (6) [38],

Hysteresis $(\%)=\frac{|\Delta T|}{T_{\max }-T_{\min }} \times 100$,

where $\Delta T$ is the maximum variation in amplitude of signal $T_{n o r m}$ for a predefined value of $R_{\text {norm }}$ and ( $T_{\max }-T_{\min }$ ) is the difference between the maximum and minimum values of $T_{\text {norm }}$. As $\left(T_{\max }-T_{\min }\right)$ is approximately equal to 1 , this term may be neglected. The sensor's response exhibited a maximum hysteresis of $1.65 \%$. As observed in Fig. 5a, the distances between the parametric curves are not constant (solid lines). The curves associated to the fiber end are closer to each other as compared with the parametric curves associated to the source end, suggesting a tradeoff between accuracy and distance. In order to demonstrate that higher spatial resolution can be achieved, losses were induced at six different regions located at $l_{i}^{\prime}=0.50,0.65,0.80,4.20,4.45,4.50 \mathrm{~m}$. The corresponding parametric curves are displayed in Fig. $5 \mathrm{a}$ as dashed lines with round and square markers, representing, respectively, disturbances induced near the source end and the fiber end. The inset in Fig. 5a shows the parametric curves obtained for $l_{i}^{\prime}=4.20,4.45,4.50 \mathrm{~m}$. Despite the curves being close to each other, the proposed approach allows to identify clearly where each disturbance occurred.

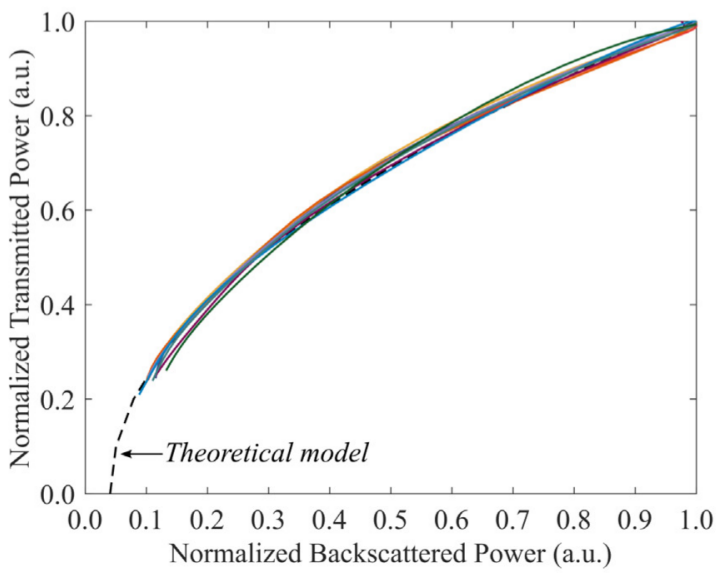

(b)

Fig. 5. Relationships between the normalized backscattered and transmitted powers for NPF (a) and SSMF (b) at different disturbance locations. Solid lines represent the measurements for $\Delta L=0.5$. Dashed lines with round and square markers represent the measurements for $\Delta L=0.15$. 


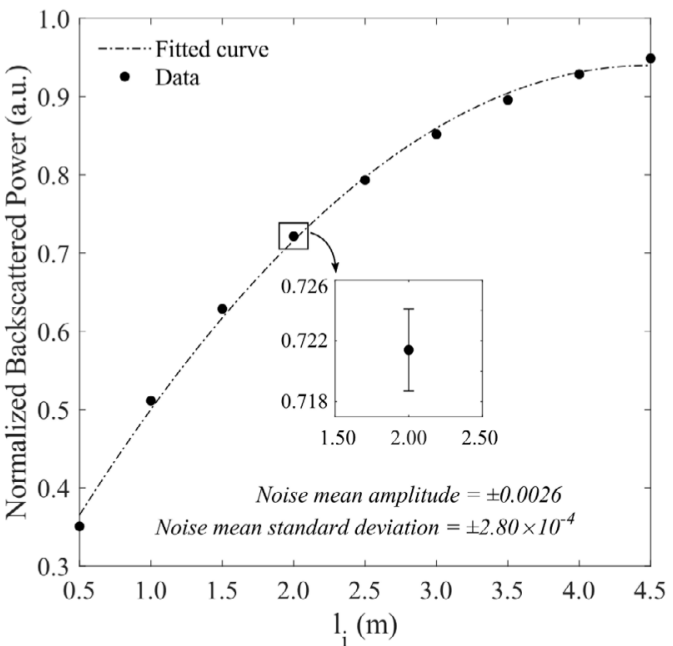

(a)

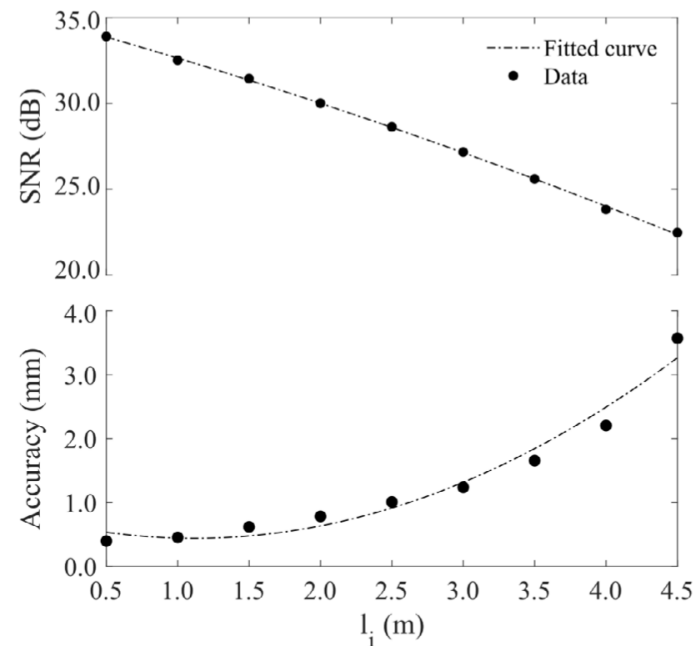

(b)

Fig. 6. Strong disturbance analysis $\left(T_{n o r m}=0.035\right)$ : normalized backscattered power (a), SNR and accuracy (b).

The TRA technique has been successfully applied to SSMF-based DOFS, whose reported lengths vary within a range of 5-21 km (Table 2). Nevertheless, the response of the 5.4-m-length sensor exhibited parametric curves which may not be visually distinct (Fig. 5b). The results obtained from the theoretical model are shown in Fig. $5 \mathrm{~b}$ as a dashed line. A good agreement between experimental data and theory was obtained for $\alpha_{s} / \alpha=0.67$. A typical value of $b=1 / 4.55$ was used $[39,14]$. In our experiment, reflections from the source-end and the remote-end were equal to $r_{1}=1.7 \times 10^{-6}$ and $r_{2}=3.9 \times 10^{-5}$, respectively. According to numerical calculations, the distance between the parametric curves is approximately constant and equal to $7.253 \times 10^{-4}$. However, during the experiment, variation in $R_{\text {norm }}$ due to noise has been estimated as $3.2 \times 10^{-3}$. Therefore, variations in the backscattered signal were too small to be captured by the photodetector [33].

\subsection{Strong and weak disturbances analyses}

In order to simulate a strong disturbance, further analyses were conducted by fixing $T_{\text {norm }}$ equal to 0.035 (the highest minimum value of $T_{\text {norm }}$ acquired at all distances $l_{i}$ ). The characterization of the normalized Rayleigh backscattered power is presented in Fig. 6. A exponential regression was performed in order to express $l i$ as a function of $R_{n o r m}$ :

$l_{i}\left(R_{\text {norm }}\right)=p_{1} e^{p_{2}\left(R_{\text {norm }}-p_{3}\right)}$

Therefore, the region where the disturbance occurred can be determined by Eq. (7) and the coefficients $p_{1}, p_{2}$ and $p_{3}$ listed in Table 1 . To analyze the response repeatability, data was recorded for $30 \mathrm{~s}$ while the micrometric manual control was kept constant. Regarding the backscattered signal, the maximum variation in amplitude due to noise was 0.0032 and the mean standard deviation was $2.8 \times 10^{-4}$. The signalto-noise ratio (SNR), which was estimated by dividing the mean value of signal $\left(1-R_{\text {norm }}\right)$ by its standard deviation, is presented in Fig. $6 \mathrm{~b}$. The SNR exhibited decreasing behavior with respect to $l_{i}$, falling more than $10 \mathrm{~dB}$ below its initial value. In the worst case, an SNR of $22.5 \mathrm{~dB}$ was obtained. Due to the non-linear behavior of light scattering in NPFs, the

Table 1

Coefficients of the exponential regression given by Eq. (7) and the corresponding R-squared error $\left(R^{2}\right)$.

\begin{tabular}{ccccc}
\hline$T_{\text {norm }}$ & $p_{1}$ & $p_{2}$ & $p_{3}$ & $R^{2}$ \\
\hline 0.035 & 0.159 & 3.484 & 0 & 0.9960 \\
0.80 & 0.16 & 18.07 & 0.80 & 0.9944 \\
\hline
\end{tabular}

system's accuracy is higher near the fiber head as compared with the fiber end. The response accuracy was estimated by the following equation,

Accuracy $=\frac{\Delta L \delta A}{\Delta R}$,

where $\Delta R$ is the difference between the normalized backscattered powers associated with distances $l_{i}$ and $l_{i+1}$ (Fig. 6a) and $\delta A$ is the maximum variation in amplitude of $R_{\text {norm }}$ due to noise. The localization accuracy for a strong disturbance has been estimated as $\pm 0.8 \mathrm{~mm}$ near the fiber head and $7.2 \mathrm{~mm}$ near the fiber end (Fig. 6b).

As depicted in Fig. 6a, the slope of $R_{\text {norm }}$ with respect to $l_{i}$ decreases with distance, coming to a moment when variations in $R_{\text {norm }}$ are very low and a tendency of saturation can be distinguished. Therefore, it is possible to conclude that the sensor is limited to a maximum length. The length analysis is conducted considering $T_{\text {norm }}$ close to zero (strongly disturbed condition) due to the excessive induced losses, which can be abstracted as a cut in fiber at distance $l_{i}$. To estimate the maximum length, the measured normalized backscattered powers (Fig. 6a) were converted into voltage and written as function of $l_{i}$ by means of an exponential regression. Variations in voltage $\left(\Delta R_{V}\right)$ between the intervals $l_{i}$ and $l_{i+1}$ were quantified with the purpose of identifying the shortest distance $l_{\max }$ associated to a value of $\Delta R_{V}$ equal or smaller than the ADC's quantization interval. Therefore, $l_{\max }$ is assumed to be the maximum length supported by the interrogation system. Since the proposed ADC has 16 bits of resolution (effectively 14 bits are available for digitization) and the MCU's analog input supports up to $3.3 \mathrm{~V}$, the maximum quantization interval is about $201.4 \mu \mathrm{V}$. Assuming $\Delta L=0.5 \mathrm{~m}$, the maximum length has been estimated as $12.1 \mathrm{~m}$. On the other hand, for $\Delta L=0.01 \mathrm{~m}, l_{\max }$ was approximately equal to $5.47 \mathrm{~m}$. It is worth noting that the NPF used in the experimental tests is $5.40 \mathrm{~m}$ in length, and, although such arbitrary length is due to availability of the fiber sample, it is within the acceptable range.

In order to examine a weakly disturbed condition, the aforementioned analysis was repeated assuming $T_{\text {norm }}$ equal to 0.8 , as introduced in [40]. Fig. 7a depicts the normalized Rayleigh backscattered power as a function of $l_{i}$. The backscattered power varied within a narrow range as compared with the previous condition of a strong disturbance, which implies lower accuracy in measurements and lower SNR. Once the backscattered power is measured, the distance $l_{i}$ can be estimated by Eq. (7) and Table 1. The mean standard deviation of $R_{\text {norm }}$ was $3.08 \times 10^{-4}$ and the maximum variation in amplitude due to noise, considering $l_{1}$ to $l_{9}$, was 0.0027 . The signal SNR varied from 26 to $17.4 \mathrm{~dB}$, as presented in Fig. $7 \mathrm{~b}$. The localization accuracy for a weak 
Table 2

Comparison of researches on TRA focused on enhancing the sensor's specifications.

\begin{tabular}{|c|c|c|c|}
\hline Paper & Length & Accuracy & Optical Medium \\
\hline [21] & $1 \mathrm{~m}$ & Accuracy not reported. Spatial resolution of $0.25 \mathrm{~m}$ & $\begin{array}{l}\text { Standard single-mode fiber with } 3 \text { FBGs inscribed into the fiber's } \\
\text { core }\end{array}$ \\
\hline [14] & $2.8 \mathrm{~km}$ & $\pm 1 \mathrm{~m}$ (strong disturbance) & Standard single-mode fiber \\
\hline [15] & $10 \mathrm{~cm}$ & $\pm 5 \mathrm{~mm}$ (weak disturbance) & $\begin{array}{l}\text { Standard single-mode fiber with } 20 \text { FBGs inscribed into the fiber's } \\
\text { core }\end{array}$ \\
\hline [15] & $3.9 \mathrm{~km}$ & $\pm 1 \mathrm{~m}$ (strong disturbance) & Standard single-mode fiber \\
\hline [12] & $21.0 \mathrm{~km}$ & $\pm 1 \mathrm{~m}$ near to the source end and $\pm 7.5 \mathrm{~m}$ near to the fiber end (strong disturbance) & Standard single-mode fiber \\
\hline This paper & $5.4 \mathrm{~m}$ & $\begin{array}{l} \pm 6.4 \mathrm{~mm} \text { near to the source end and } \pm 36.7 \mathrm{~mm} \text { near the fiber end (weak } \\
\text { disturbance) }\end{array}$ & Magnesium and erbium co-doped fiber \\
\hline
\end{tabular}

disturbance has been estimated as $\pm 6.4 \mathrm{~mm}$ near the fiber head and $36.7 \mathrm{~mm}$ near the fiber end (Fig. $7 \mathrm{~b}$ ).

Table 2 presents the outcome of researches focused on enhancing the specifications of TRA-based DOFS. As far as the authors know, the highest accuracy achieved with the method is $\pm 5 \mathrm{~mm}$ [15]. However, this result comes from the inscription of 20 FBGs into the core of a 10cm-length SSMF, which requires complex setups and potentially affects the sensor's robustness. It is worth noting that, if longer lengths were required, more gratings would be needed in order to preserve accuracy: a 1-m-length DOFS with an accuracy of $\pm 5 \mathrm{~mm}$ would demand 200 inscribed FBGs. On the other hand, pure SSMF sensors are limited to applications of long distances (see Table 2). As previously mentioned, this methodology yielded inconclusive results for a 5.4-m-length fiber.

The use of NPF as optical medium dismisses complex setups required for inscribing hundreds of FBGs and offers an accuracy up to tens of millimeters. Nevertheless, TRA-based NPF sensors are limited to short-range applications due to high attenuation in fiber caused by scattering losses. Hence, applications of this nature that require to track a single perturbation can be further explored, such as: smart carpets for gait analysis [41], temperature and strain sensing systems for structural health monitoring [13] and shape sensing systems for smart structure monitoring [42]. Moreover, as the proposed system requires just electronic and passive optical devices, it is possible to create compact-size interrogators for wearable applications, such as robotic exoskeletons for human-robot forces assessment [43-45] and smart textiles for sports motion and vital signs monitoring [46,47]. Regarding impact detection, many conventional distributed monitoring systems have low sampling rates $[48,49]$. Thus, the proposed approach may be a potential solution for impact detection systems running up to hundreds of $\mathrm{MHz}$. In addition, since the NPF sensor employs a standard MCU, IoT modules can be easily connected for smart-home and smart-cities applications [50,51].

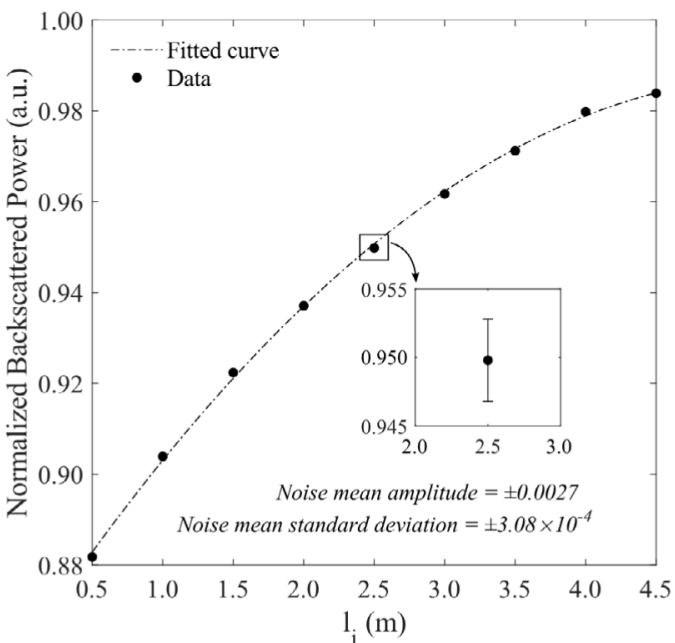

(a)

\section{Conclusions}

This paper presented experimental evidence that inexpensive distributed optical fiber sensors can be produced by combining the wellstabilised TRA method with a high scattering optical fiber. We have demonstrated that the proposed approach exhibits higher spatial resolution than its counterpart, the SSMF-based sensor. Experimental tests have shown that the proposed sensor can be used to locate bending losses with a spatial resolution of $0.5 \mathrm{~m}$ and $0.15 \mathrm{~m}$. The localization of a strong disturbance with an estimated accuracy of up to $\pm 0.8 \mathrm{~mm}$ near the source end and $\pm 7.2 \mathrm{~mm}$ near the fiber end was analytically demonstrated. In regards to weak disturbances, the response accuracy has been estimated up to $\pm 6.4 \mathrm{~mm}$ near the source and $\pm 36.7 \mathrm{~mm}$ near the fiber end. The variations in accuracy along the sensor's length come from the fact that SNR decreases as the deformation event moves toward the fiber end. Spatial resolution and accuracy can be considerably improved by increasing the ADC's resolution. Hardware modifications and more detailed repeatability analysis will be addressed in future work in order to improve the sensor setup and specifications. Moreover, the temperature cross-sensitivity and the effect of mechanical disturbances on the sensor performance will be investigated in future work.

\section{CRediT authorship contribution statement}

Mariana Silveira: Validation, Formal analysis, Investigation, Data curation, Writing - original draft. Anselmo Frizera: Methodology, Writing - review \& editing. Arnaldo Leal-Junior: Conceptualization, Writing - review \& editing. Diana Ribeiro: Formal analysis, Writing review \& editing. Carlos Marques: Visualization, Writing - review \& editing. Wilfried Blanc: Resources, Writing - review \& editing. Camilo

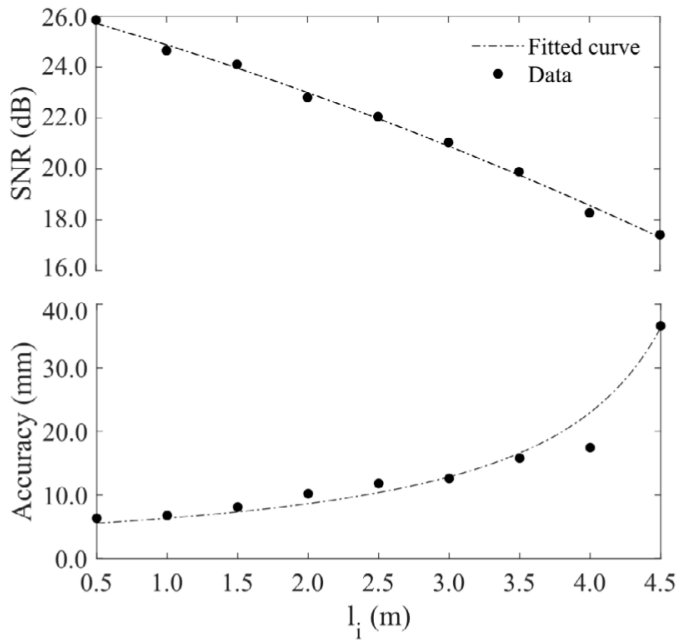

(b)

Fig. 7. Weak disturbance analysis $\left(T_{\text {norm }}=0.8\right)$ : normalized backscattered power (a), SNR and accuracy (b). 
A. R. Díaz: Conceptualization, Methodology, Validation, Investigation, Writing - review \& editing, Supervision.

\section{Declaration of Competing Interest}

The authors declare that they have no known competing financial interests or personal relationships that could have appeared to influence the work reported in this paper.

\section{Acknowledgement}

The authors acknowledge S. Trzesien and M. Ude (INPHYNI, Nice, France) for the fabrication of the fiber sample. This project was supported in part by Petrobras S.A. under Grant 2017-00702/6, in part by CAPES-financing code 001, in part by CNPq:304049/2019-0 under Grant 408480/2018-1 and in part by FAPES under Grant 84336650. C. Marques acknowledges Fundação para a Ciência e a Tecnologia (FCT) through the CEECIND/ 00034/2018 (iFish project). This work was developed within the scope of the project i3N, UIDB/50025/2020 \& UIDP/50025/2020, financed by national funds through the FCT/MEC. This project is also supported by Agence Nationale de la Recherche (ANR) (ANR-17-CE08-0002) and National Funds (OE), through FCT, I.P., in the scope of the framework contract foreseen in the numbers 4, 5 and 6 of the article 23, of the Decree-Law 57/2016, of August 29, changed by Law 57/2017, of July 19.

\section{References}

[1] A.G. Hartog, D.N. Payne, Remote measurement of temperature distribution using an optical fibre, in: 8th ECOC, no. July, Cannes, France, 1982, pp. 215-220.

[2] A.H. Hartog, A.P. Leach, M.P. Gold, Distributed temperature sensing in solid-core fibres, Electronics Letters 21 (23) (1985) 1061-1062, https://doi.org/10.1049/ el: 19850752.

[3] B. Culshaw, Optical fibre sensors: a current perspective, The Open Optics Journal 7 (1) (2014) 21-31, https://doi.org/10.2174/1874328501307010021.

[4] B.H. Lee, Y.H. Kim, K.S. Park, J.B. Eom, M.J. Kim, B.S. Rho, H.Y. Choi, Interferometric fiber optic sensors, Sensors 12 (3) (2012) 2467-2486, https://doi. org/10.3390/s120302467.

[5] L. Zhang, L.D. Costa, Z. Yang, M.A. Soto, M. Gonzalez-Herraez, L. Thevenaz, Analysis and reduction of large errors in rayleigh-based distributed sensor, Journal of Lightwave Technology 37 (18) (2019) 4710-4719, https://doi.org/10.1109/JLT. 2019.2917746.

[6] A. Hartog, Distributed fiber-optic sensors: principles and applications, in: K.T.V. Grattan, B.T. Meggitt (Eds.), Optical Fiber Sensor Technology, Kluwer Academic Publishers, Boston, United States, 2000, pp. 241-301.

[7] A. Barrias, G. Rodriguez, J.R. Casas, S. Villalba, Application of distributed optical fiber sensors for the health monitoring of two real structures in Barcelona, Structure and Infrastructure Engineering 14 (7) (2018) 967-985, https://doi.org/10.1080/ 15732479.2018.1438479.

[8] A. Barrias, J.R. Casas, S. Villalba, Fatigue performance of distributed optical fiber sensors in reinforced concrete elements, Construction and Building Materials 218 (2019) 214-223, https://doi.org/10.1016/j. conbuildmat.2019.05.072.

[9] L. Ren, T. Jiang, Z. guang Jia, D. sheng Li, C. lin Yuan, H. nan Li, Pipeline corrosion and leakage monitoring based on the distributed optical fiber sensing technology, Measurement: Journal of the International Measurement Confederation 122 (2018) 57-65. doi: 10.1016/j.measurement.2018.03.018.

[10] I. Laarossi, M.Á. Quintela-Incera, J.M. López-Higuera, Comparative experimental study of a high-temperature raman-based distributed optical fiber sensor with different special fibers, Sensors 19 (3) (2019) 1-13, https://doi.org/10.3390/ s19030574.

[11] D. Wada, H. Igawa, M. Tamayama, T. Kasai, H. Arizono, H. Murayama, N. Isoda, M. Katsuta, Fiber-optic simultaneous distributed monitoring of strain and temperature for an aircraft wing during flight, Applied Optics 57 (36) (2018) 10458-10465, https://doi.org/10.1364/AO.57.010458.

[12] V.V. Spirin, P.L. Swart, S.V. Miridonov, 20-km-length distributed fiber optical loss sensor based on transmission-reflection analysis, Optical Engineering 44 (4) (2005) 20-21, https://doi.org/10.1117/1.1883585.

[13] M. Zhang, T. Wang, Temperature and crack measurement using distributed opticfiber sensor based on raman loop configuration and fiber loss temperature and crack measurement using distributed optic-fiber sensor based on raman loop configuration, IEEE Photonics Journal 11 (4) (2019) 1-13, https://doi.org/10.1109/JPHOT. 2019.2931306.

[14] R.M. Lopez, V.V. Spirin, S.V. Miridonov, M.G. Shlyagin, G. Beltrán, E.A. Kuzin, Fiber optic distributed sensor for hydrocarbon leak localization based on transmission/reflection measurement, Optics \& Laser Technology 34 (6) (2002) 465-469, https://doi.org/10.1016/S0030-3992(02)00043-9.

[15] V.V. Spirin, F.J. Mendieta, S.V. Miridonov, M.G. Shlyagin, A.A. Chtcherbakov,
P.L. Swart, Localization of a loss-inducing perturbation with variable accuracy along a test fiber using transmission-reflection analysis, IEEE Photonics Technology Letters 16 (2) (2004) 2003-2005.

[16] M.K. Barnoski, D.M. Rourke, S.M. Jensen, R.T. Melville, Optical time domain reflectometry, Applied Optics 16 (9) (1977) 2375-2379.

[17] W. Eickhoff, R. Ulrich, Optical frequency domain reflectometry in single-mode fiber, Applied Physics Letters 39 (9) (1981) 693-695, https://doi.org/10.1063/1. 92872.

[18] M. Cen, V. Moeyaert, P. Mégret, M. Wuilpart, Localization and quantification of reflective events along an optical fiber using a bi- directional TRA technique, Optics Express 22 (8) (2014) 9839-9853, https://doi.org/10.1364/OE.22.009839.

[19] X. Liu, B. Jin, Q. Bai, Y. Wang, D. Wang, Y. Wang, Distributed fiber-optic sensors for vibration detection, Sensors 16 (8) (2016) 1-31, https://doi.org/10.3390/ s16081164.

[20] A.G. Leal-Junior, C.A. Diaz, L.M. Avellar, M.J. Pontes, C. Marques, A. Frizera, Polymer optical fiber sensors in healthcare applications: A comprehensive review, Sensors 19 (14) (2019) 1-30, https://doi.org/10.3390/s19143156.

[21] V.V. Spirin, M.C. Shlyagin, S.V. Miridonov, P.L. Swart, Transmission/reflection analysis for distributed optical fibre loss sensor interrogation, Eletronics Letters 38 (3) (2002) 117-118, https://doi.org/10.1049/el:20020096.

[22] J. Li, J. Gan, Z. Zhang, X. Heng, C. Yang, Q. Qian, S. Xu, Z. Yang, High spatial resolution distributed fiber strain sensor based on phase-OFDR, Optics Express 25 (22) (2017) 27913, https://doi.org/10.1364/oe.25.027913.

[23] S. Loranger, M. Gagné, V. Lambin-Iezzi, R. Kashyap, Rayleigh scatter based order of magnitude increase in distributed temperature and strain sensing by simple UV exposure of optical fibre, Scientific Reports 5 (2015) 11177, https://doi.org/10. 1038/srep 11177.

[24] L. Pereira, R. Min, X. Hu, C. Caucheteur, O. Bang, B. Ortega, C. Marques, P. Antunes, J.L. Pinto, Polymer optical fiber Bragg grating inscription with a single Nd:YAG laser pulse, Optics Express 26 (14) (2018) 18096, https://doi.org/10.1364/oe.26. 018096.

[25] C.A. Marques, A.G. Leal-Junior, R. Min, M. Domingues, C. Leitão, P. Antunes, B. Ortega, P. André, Advances on polymer optical Fiber Gratings using a KrF pulsed laser system operating at $248 \mathrm{~nm}$, Fibers 6 (1) (2018) 1-13, https://doi.org/10. 3390/fib6010013.

[26] C.A. Díaz, C. Leitão, C.A. Marques, M.F. Domingues, N. Alberto, M.J. Pontes, A. Frizera, M.R. Ribeiro, P.S. André, P.F. Antunes, Low-cost interrogation technique for dynamic measurements with FBG-based devices, Sensors 17 (10) (2017) 1-10, https://doi.org/10.3390/s17102414.

[27] A. Veber, Z. Lu, M. Vermillac, F. Pigeonneau, W. Blanc, L. Petit, Nano-Structured optical fibers made of glass-ceramics, and phase separated and metallic particlecontaining glasses, Fibers 7 (12) (2019) 105, https://doi.org/10.3390/fib7120105.

[28] W. Blanc, B. Dussardier, Formation and applications of nanoparticles in silica optical fibers, Journal of Optics 45 (September) (2016) 247-254, https://doi.org/10 1007/s12596-015-0281-6.

[29] A. Beisenova, A. Issatayeva, S. Korganbayev, C. Molardi, W. Blanc, D. Tosi, Simultaneous distributed sensing on multiple MgO-doped high scattering fibers by means of scattering-level multiplexing, Journal of Lightwave Technology 37 (13) (2019) 3413-3421, https://doi.org/10.1109/JLT.2019.2916991.

[30] A. Beisenova, A. Issatayeva, I. Iordachita, W. Blanc, C. Molardi, D. Tosi, Distributed fiber optics 3D shape sensing by means of high scattering NP-doped fibers simultaneous spatial multiplexing, Optics Express 27 (16) (2019) 22074, https://doi. $\mathrm{org} / 10.1364 / 0 \mathrm{o} .27 .022074$.

[31] M. Sypabekova, S. Korganbayev, W. Blanc, T. Ayupova, A. Bekmurzayeva, M. Shaimerdenova, K. Dukenbayev, C. Molardi, D. Tosi, Fiber optic refractive index sensors through spectral detection of Rayleigh backscattering in a chemically etched MgO-based nanoparticle-doped fiber, Optics Letters 43 (24) (2018) 5945, https://doi.org/10.1364/ol.43.005945.

[32] S. Korganbayev, M. Shaimerdenova, T. Ayupova, M. Sypabekova, A. Bekmurzayeva, W. Blanc, C. Molardi, D. Tosi, Refractive index sensor by interrogation of etched $\mathrm{MgO}$ nanoparticle-doped optical fiber signature, IEEE Photonics Technology Letters 31 (15) (2019) 1253-1256, https://doi.org/10.1109/LPT.2019.2924652.

[33] V.V. Spirin, Transmission/reflection analysis for localization of temporally successive multipoint perturbations in a distributed fiber-optic loss sensor based on Rayleigh backscattering, Applied Optics 42 (7) (2003) 1175-1181, https://doi.org/ 10.1364/AO.42.001175

[34] W. Blanc, V. Mauroy, L. Nguyen, B.N.S. Bhaktha, P. Sebbah, B.P. Pal, B. Dussardier, Fabrication of rare earth-doped transparent glass ceramic optical fibers by modified chemical vapor deposition, Journal of the American Ceramic Society 94 (8) (2011) 2315-2318, https://doi.org/10.1111/j.1551-2916.2011.04672.x.

[35] W. Blanc, I. Martin, H. Francois-Saint-Cyr, X. Bidault, S. Chaussedent, C. Hombourger, S. Lacomme, P. Le Coustumer, D.R. Neuville, D.J. Larson, T.J. Prosa, C. Guillermier, Compositional changes at the early stages of nanoparticles growth in glasses, Journal of Physical Chemistry C 123 (47) (2019) 29008-29014, https://doi.org/10.1021/acs.jpcc.9b08577.

[36] M. Vermillac, J.F. Lupi, F. Peters, M. Cabié, P. Vennéguès, C. Kucera, T. Neisius, J. Ballato, W. Blanc, Fiber-draw-induced elongation and break-up of particles inside the core of a silica-based optical fiber, Journal of the American Ceramic Society 100 (5) (2017) 1814-1819, https://doi.org/10.1111/jace.14774.

[37] V. Lebihan, A. Pillonnet, D. Amans, G. Ledoux, O. Marty, C. Dujardin, Critical dimension where the macroscopic definition of refractive index can be applied at a nanometric scale, Physical Review B: Condensed Matter and Materials Physics 78 (11) (2008), https://doi.org/10.1103/PhysRevB.78.113405.

[38] A.G. Leal-Junior, A. Frizera, M. José Pontes, Sensitive zone parameters and curvature radius evaluation for polymer optical fiber curvature sensors, Optics and LaserTechnology 100 (2018) 272-281, https://doi.org/10.1016/j.optlastec.2017. 
10.006.

[39] J. Beller, Otdrs and backscatter measurements, in: D. D (Ed.), Fiber optic test and measurement, Prentice-Hall, Upper Saddle River, NJ, 1998.

[40] C.A. Rodríguez, M.R. Ribeiro, A. Frizera, M.J. Pontes, Interrogation of optical fiber based on the fusion of OFDR and TRA techniques, Optical and Quantum Electronics 48 (4) (2016) 1-6, https://doi.org/10.1007/s11082-016-0501-6.

[41] L.M. Avellar, A.G. Leal-Junior, C.A. Diaz, C. Marques, A. Frizera, POF smart carpet: A multiplexed polymer optical fiber-embedded smart carpet for gait analysis, Sensors 19 (15) (2019) 1-11, https://doi.org/10.3390/s19153356.

[42] J. Zhang, G. Sun, H. Li, X. Lou, L. Zhu, Optical fiber shape sensing of polyimide skin for flexible morphing wing, Yi Qi Yi Biao Xue Bao/Chinese Journal of Scientific Instrument 39 (2) (2018) 66-72, https://doi.org/10.19650/j.cnki.cjsi.j1702559.

[43] A.G. Leal-Junior, A. Frizera-Neto, M.J. Pontes, T.R. Botelho, Hysteresis compensation technique applied to polymer optical fiber curvature sensor for lower limb exoskeletons, Measurement Science and Technology 28 (12) (2017) 1-10, https:// doi.org/10.1088/1361-6501/aa946f.

[44] A.G. Leal-Junior, A. Frizera, L.M. Avellar, M.J. Pontes, Design considerations, analysis, and application of a low-cost, fully portable, wearable polymer optical fiber curvature sensor, Applied Optics 57 (24) (2018) 6927, https://doi.org/10. 1364/ao.57.006927.

[45] A.G. Leal-Junior, C.R. Díaz, M.J. Pontes, C. Marques, A. Frizera, Polymer optical fiber-embedded, 3D-printed instrumented support for microclimate and humanrobot interaction forces assessment, Optics and Laser Technology 112 (2019)
323-331, https://doi.org/10.1016/j.optlastec.2018.11.044.

[46] J. Zhang, Y. Cao, M. Qiao, L. Ai, K. Sun, Q. Mi, S. Zang, Y. Zuo, X. Yuan, Q. Wang, Human motion monitoring in sports using wearable graphene-coated fiber sensors, Sensors and Actuators, A: Physical 274 (2018) 132-140, https://doi.org/10.1016/j. sna.2018.03.011.

[47] Y. Koyama, M. Nishiyama, K. Watanabe, Smart textile using hetero-core optical fiber for heartbeat and respiration monitoring, IEEE Sensors Journal 18 (15) (2018) 6175-6180, https://doi.org/10.1109/JSEN.2018.2847333.

[48] D. Vilarinho, A. Theodosiou, C. Leitão, A.G. Leal-Junior, M. de Fátima Domingues, K. Kalli, P. André, P. Antunes, C. Marques, POFBG-embedded cork insole for plantar pressure monitoring, Sensors 17 (12) (2017) 1-15, https://doi.org/10.3390/ s17122924.

[49] R. Suresh, S. Bhalla, J. Hao, C. Singh, Development of a high resolution plantar pressure monitoring pad based on fiber Bragg grating (FBG) sensors, Technology and Health Care 23 (6) (2015) 785-794, https://doi.org/10.3233/THC-151038.

[50] G. Feng, J. Mai, Z. Ban, X. Guo, G. Wang, Floor pressure imaging for fall detection with fiber-optic sensors, IEEE Pervasive Computing 15 (2) (2016) 40-47, https:// doi.org/10.1109/MPRV.2016.27.

[51] Y. Huang, P. Lu, R. Bridgelall, Road sensor network for smart city applications, in: Sensors and Smart Structures Technologies for Civil, Mechanical, and Aerospace Systems 2018, no. 10598, Denver, United States, 2018, p. 125. doi:10.1117/12. 2295949. 